ARTÍCULOS ORIGINALES Rev Chil Salud Pública 2014; Vol 18 (3): 263-273

\section{DETERMINACIÓN DE RESIDUOS DE PLAGUICIDAS EN TRABAJADORES AGRÍCOLAS DEL MUNICIPIO DE BARCELONA, QUINDÍO- COLOMBIA}

\author{
DETERMINATION OF PESTICIDE LEVELS IN FARMERS WORKING IN THE \\ Barcelona municipality, QUindio, Colombia
}

\section{RESUMEN}

Objetivos: En este trabajo se determinaron los niveles de residualidad de plaguicidas organoclorados y organofosforados en muestras de sangre periférica de trabajadores agrícolas -fumigadores-en el municipio de Barcelona, Quindio, Colombia.

Método: Los trabajadores fueron escogidos al azar dentro de los principales cultivos abastecedores de frutas y verduras de la región. Para la extracción y determinación de los analitos en sangre, se usó la técnica de micro-extracción en fase sólida en modo head space y cromatografía de gases capilar dotado con un detector de micro captura de electrones.

Resultados: Como resultado se encontraron residuos de 21 plaguicidas de tipo organoclorados y organofosforados; la mayoría de estos en alta concentración (>0.01 ppm) y probibidos por la legislación nacional e internacional, sugiriendo que aún siguen siendo utilizados ilegalmente. De la residualidad encontrada, los plaguicidas más representativos fueron: beta-BHC y endosulfan, ballados en el $50 \%$ de las personas evaluadas. Asimismo, se descubrió que entre el 20 y 40\% de las personas tenian residuos de endrin aldehido, forato, sulfotep, disulfoton y thionazin, en circunstancia que la mayoría de estos son de categoría toxicología I y II, comprobando el alto riesgo al que se exponen constantemente los individuos que aplican estos compuestos y la falta de atención e información por parte de las entidades responsables de autorización y fiscalización de su uso.

Conclusiones: En este sentido, los resultados encontrados en este trabajo demuestran la importancia de continuar con los estudios de monitoreo y control de calidad de los principales alimentos, fuentes hídricas y trabajadores agrícolas, para así informar a la población acerca de los riesgos a los que está expuesta.

Palabras clave: plaguicidas, organoclorados, organofosforados, residuos, sangre, trabajadores agrícolas. 


\section{ABSTRACT}

Aim: To determine the levels of organochlorine and organophosphorus pesticide residues in peripheral blood samples of farm-workers in Barcelona, Quindio, Colombia.

Methods: Farm-workers were randomly chosen to cover the major suppliers of fruits and vegetables crops in the region. Solid phase micro extraction in head space mode and capillary gas chromatography with a micro electron capture detector were used for extraction and determination of analytes from blood.

Results: Twenty-one pesticides residues among organochlorine and organophosphorusclasses were found. Most of these pesticides were at high concentrations ( $>0.01 \mathrm{ppm})$ andrepresented concentrations forbidden by national and international legislation. Beta-BHC and endosulfan were found in $50 \%$ of persons tested. Residues of endrinaldebyde, phorate, sulfotep, disulfoton and thionazinwere found amongst $20-40 \%$ of the persons tested.

Conclusions: These results demonstrate the high risks that farm-workers are exposed to, suggesting poor attention and provision of information byentities responsible for permits and supervision. Furthermore, we highlight theneed for monitoring and quality control of food, water sources and farm-workers, and the necessity to inform workers and the community of the risks to which they are exposed.

Key words: Pesticides, organochlorine, organophosphorus, residues, blood, farm-workers.

\section{INTRODUCCIÓN}

En países tropicales como Colombia la introducción de plaguicidas en la agricultura y en los programas sanitarios, proporcionó grandes beneficios en el control de plagas y en la propagación de enfermedades transmitidas por vectores y ectoparásitos. Debido a su bajo costo este tipo de productos químicos son accesibles y utilizados ampliamente en prácticas agrícolas. ${ }^{1}$ Como consecuencia, muchas personas entran en contacto con estos compuestos, exponiéndose a elementos químicos no deseados, ya sea por consumo de alimentos, por contaminación de su hábitat o el lugar de trabajo. ${ }^{2}$ La residualidad en el cuerpo humano se debe a la naturaleza lipofílica de los plaguicidas, que tienden a acumularse en múltiples compartimientos del organismo. ${ }^{3-6}$ Según el sistema de vigilancia en salud pública (Sivigila), en Colombia cada año aumenta el número de casos de intoxicación aguda por plaguicidas, llegando a 8016 intoxicados en el $2010 .{ }^{4}$ Esto es debido tanto al sistema de propagación que tienen los plaguicidas una vez aplicados, como al uso inadecuado de los mismos, ${ }^{5}$ ya que en la ma- yoría de los casos predomina el aumento de la producción de los cultivos, subestimando las posibles consecuencias que esto conlleva; generando malas prácticas agrícolas -como el uso indiscriminado de plaguicidas-. Lo anterior puede ocasionar serios problemas a la salud de la población expuesta. ${ }^{6,7}$

Si bien el uso de plaguicidas mejora satisfactoriamente la producción de alimentos, la exposición a estos compuestos está asociada a un creciente número de efectos agudos y crónicos en la salud. ${ }^{3,8-10}$ Por lo tanto, el monitoreo y control de residuos de plaguicidas en alimentos y agricultores es de gran importancia para minimizar la exposición de los consumidores y mejorar las condiciones de trabajo de los trabajadores agrícolas. ${ }^{9-12}$

A pesar de que se conoce la contaminación de plaguicidas Organoclorados "OCs" (alfa BHC, heptaclor, delta y gama BHC y 4,4 DDT) y Organofosforados "OFs" (endosulfan I y II, endosulfan sulfato, aldrin) en ríos, quebradas y suelos de diferentes zonas del departamento del Quindío, ${ }^{10,11}$ es poco el conocimiento de la residualidad de estos productos en los aplicadores. En este sentido, existe la necesidad de realizar estudios en las 
personas directamente implicadas en la fumigación de los cultivos, con el fin de determinar la posible residualidad, la cual pudiera originarse en el uso inadecuado de los agroquímicos y/o de los implementos de protección personal. Conocer los niveles reales de estos compuestos en el organismo permitirá generar conciencia acerca de las posibles consecuencias en la salud. ${ }^{12,13}$

Últimamente se ha generado un interés científico en los posibles efectos adversos para la salud de la exposición a plaguicidas, forjando la necesidad de nuevos métodos analíticos para la medición de los marcadores de exposición a estos compuestos. ${ }^{6} \mathrm{La}$ cromatografía de gases GC (por sus siglas en inglés Gas Chromatography) es una técnica de separación ampliamente usada, debido a la sensibilidad y selectividad de los detectores con los que cuenta, como el detector de captura de electrones ECD (por sus siglas en inglés Electron Capture Detector) y detector de nitrógeno fósforo NPD (por sus siglas en inglés Nitrogen Phosphorus Detector), entre otros. ${ }^{14,15}$

En este sentido, para determinar la posible residualidad de plaguicidas OCs y OFs en la sangre de trabajadores agrícolas, se utilizó la extracción en fase sólida o SPE (por sus siglas en inglés Solid Phase Extraction), en conjunto con cromatografía de gases y un detector de microcaptura de electrones $(\mu-E C D) .{ }^{16-18}$

\section{MATERIALES Y MÉTODOS}

Se realizó un estudio descriptivo a 42 trabajadores agrícolas, encargados de la fumigación en la mayoría de los cultivos de la zona "Agua Bonita” en el departamento del Quindío. En primera instancia los trabajadores fueron reclutados e incluidos por medio de un consentimiento informado, instancia en la cual se les puso al tanto sobre la investigación y el requerimiento de muestras de sangre de manera voluntaria. Asimismo, se realizó una encuesta a cada fumigador, donde se registraron datos personales y ocupacionales.

Se tomaron muestras de $5 \mathrm{ml}$ de sangre periférica en un tubo vacutainer tapón rojo, las cuales se dejaron en reposo por $30 \mathrm{~min}$ para permitir una coagulación efectiva. Posteriormente se centrifugaron las muestras por $10 \mathrm{~min}$ a $2500 \mathrm{rpm}$ y se extrajeron los sueros lipídicos, los cuales se conservaron a $-20^{\circ} \mathrm{C}$ hasta su extracción y análisis cromatográfico. La extracción de los plaguicidas en las muestras de suero se realizó por microextracción en fase sólida (SPME) a 50 ${ }^{\circ} \mathrm{C}$ durante 30 min bajo agitación magnética. Para la evaluación de los residuos de plaguicidas se utilizó un cromatógrafo de gases Hewlett-Packard HP 6890N dotado con un detector de microcaptura de electrones $(\mu-E C D)$ y una columna Supelco Equity-5 $(0,25 \mathrm{~mm}$ I.D $\times 0,25 \mu \mathrm{m}$ film $\times 30 \mathrm{~m})$. Las temperaturas del inyector y detector permanecieron a 250 y $300{ }^{\circ} \mathrm{C}$ respectivamente, siguiendo la rampa de calentamiento validada por nuestro grupo. ${ }^{9}$ Los resultados obtenidos de todos los estudios, tanto de laboratorio como ocupacionales, fueron procesados en un programa de cálculo convencional (origin 8.0).

\section{RESULTADOS}

\section{Población estudiada}

Como se puede observar en la Tabla 1, el rango de edades es amplio: se estudiaron personas menores de 20 y mayores de 75 años. Según los testimonios brindados por los trabajadores, es una labor que se inicia a temprana edad y donde la mayoría abandona sus estudios para contribuir a sostener a su familia, labor en que permanecen por décadas.

\section{Resultados bioquímicos}

La extracción de los analitos por SPMEhs en la matriz lipídica resultó satisfactoria; los cromatogramas resultaron "claros", con picos bien definidos y líneas base influctuantes, a pesar de la complejidad de la muestra.

Se realizaron los estudios analíticos a las 42 muestras, encontrándose niveles elevados de plaguicidas en el $100 \%$ de las personas implicadas en este estudio. La mayoría de los compuestos fueron organoclorados de 
alta toxicidad, tal cual lo muestra la Figura 1, que corresponde a un trabajador escogido al azar.

Como se puede ver en el cromatograma de la figura 1 , hay una alta residualidad de plaguicidas tanto OCs como OFs. En este cromatograma en particular se destaca el beta-BHC, un compuesto organoclorado altamente tóxico que generó un pico dominante a un tiempo de retención corto. En general todas las muestras arrojaron residualidad, unas en menor cantidad, otras en grandes concentraciones, pero ya el solo hecho de que ninguna persona evaluada esté libre de residuos es preocupante. En este sentido la Tabla 2 muestra las concentraciones mínimas y máximas encontradas, la media estadística y el porcentaje poblacional afectado por estos residuos.

Tabla 1. Datos demográficos de los trabajadores agrícolas evaluados

\begin{tabular}{lc}
\hline \multicolumn{2}{c}{ Edad de trabajadores agrícolas } \\
\hline Mínima & 19 \\
Máxima & 78 \\
Promedio \pm DS & $45 \pm 15.8$
\end{tabular}

\begin{tabular}{lc} 
Número de personas & \\
Entre 18-30 años & 10 \\
Entre 31-50 años & 15 \\
Entre 51-80 años & 17 \\
Masculino & 36 \\
Femenino & 6 \\
\hline
\end{tabular}

Figura 1. Cromatograma de residuos de plaguicidas encontrados en una de las muestras de suero analizadas.

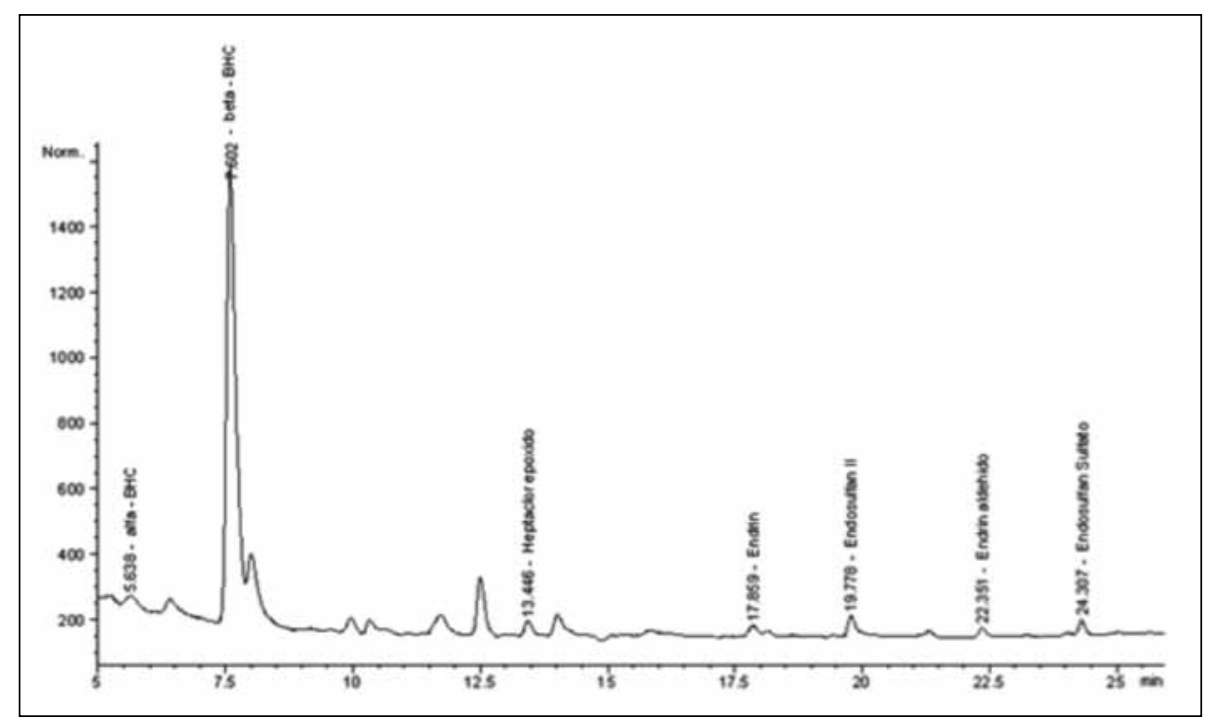


Como se puede observar en la tabla, el $50 \%$ de la población estudiada presenta residuos de beta-BHC y endosulfan II, entre el 20 y el $25 \%$ endrin aldehído, endosulfan I y alfa-BHC y por debajo del $20 \%$ residuos de otros 10 plaguicidas OCs. Estas cifras son críticas desde diferentes puntos de vista. Desde el punto de vista legal el beta-BHD es un isómero del hexaclorociclohexano $(\mathrm{HCH})$ y subproducto de la producción de lindano, un insecticida prohibido por legislación internacional hace más de 25 años. ${ }^{17}$ Por otra parte, a nivel toxicológico, es un compuesto neurotóxico que causa estrés oxidativo y afecta el sistema dopaminérgico, mostrando relación con enfermedades como el mal de Parkinson. ${ }^{18}$ Por su parte, el endosulfan es un disruptor endocrino altamente tóxico, que causa daños reproductivos y en el desarrollo en animales y humanos, prohibido en más de 50 países y categorizado por la EPA (Environmental Protection Agency) en categoría I, "altamente toxico", ${ }^{19}$ afecta enzimas involucradas en la transferencia de impulsos nerviosos..$^{20}$

Asimismo, en la Tabla 2 se muestra el porcentaje poblacional -fumigadores- que presentan residuos de plaguicidas OFs. Se encontró que más del $30 \%$ de las personas presentan residuos de forato, disulfaton y sulfotep; resultado preocupante teniendo en cuenta que el forato es el OFs más tóxico que

Tabla 2. Residuos de plaguicidas organoclorados y organofosforados encontrados en la sangre de trabajadores agrícolas

\begin{tabular}{|c|c|c|c|c|}
\hline Plaguicidas & $\begin{array}{c}\text { Concentración mínima } \\
\text { encontrada (ppm) }\end{array}$ & $\begin{array}{c}\text { Concentración máxima } \\
\text { encontrada }(\mathrm{ppm})\end{array}$ & Media & $\%$ de la población afectada \\
\hline \multicolumn{5}{|l|}{ Organoclorados } \\
\hline Beta - BHC & 0,0002 & 0,136 & 0,047 & 50 \\
\hline Heptacloro epóxido & 0,0005 & 0,009 & 0,003 & 7,1 \\
\hline Endosulfan II & 0,001 & 0,036 & 0,007 & 50 \\
\hline Endrin Aldehído & 0,001 & 0,019 & 0,005 & 26,2 \\
\hline 4,4-DDE & 0.002 & 0.003 & 0,002 & 14.3 \\
\hline Dieldrin & 0.001 & 0.009 & 0,003 & 14.3 \\
\hline Endosulfan I & 0.0001 & 0.014 & 0,004 & 23.8 \\
\hline 4,4 DDD & 0.0002 & 0.003 & 0,001 & 14.3 \\
\hline 4,4 DDT & 0.0004 & 0.006 & 0,002 & 14.3 \\
\hline Aldrin & 0.0007 & 0.031 & 0,007 & 11.9 \\
\hline Alfa BHC & 0.0004 & 0.008 & 0,002 & 19 \\
\hline Delta BHC & 0.003 & 0.004 & 0,003 & 4.8 \\
\hline Sigma BHC & 0.002 & 0.002 & 0,002 & 7.1 \\
\hline Heptacloro & 0.0003 & 0.013 & 0,005 & 14.3 \\
\hline Endrin & 0.0005 & 0.005 & 0,001 & 14.3 \\
\hline \multicolumn{5}{|l|}{ Organofosforados } \\
\hline Thionazin & 0.002 & 0.2 & 0,121 & 23.8 \\
\hline Forato & 0.003 & 3.069 & 0,535 & 33.3 \\
\hline Dimetoato & 0.002 & 0.015 & 0,006 & 7.1 \\
\hline Disulfoton & 0.02 & 0.752 & 0,097 & 38.1 \\
\hline Metilparation & 0.002 & 1.037 & 0,168 & 16.6 \\
\hline Sulfotep & 0.113 & 5.518 & 2.343 & 30.9 \\
\hline
\end{tabular}


se conoce, con una DL50 de $1-5 \mathrm{mg} / \mathrm{Kg}$ que genera persistencia en el ambiente y animales gracias a sus enlaces $\mathrm{P}-\mathrm{S}$ y $\mathrm{P}=\mathrm{S}$, es inhibidor de la enzima acetilcolinesterasa, ${ }^{21}$ una enzima de la familia de colinesterasas que se encuentra en los tejidos nerviosos y los glóbulos rojos, cuya función principal es hidrolizar al neurotransmisor acetilcolina. ${ }^{22} \mathrm{El}$ mismo efecto causa el sulfotep, otro de los fitosanitarios encontrado en el $30 \%$ de los fumigadores evaluados.

Cabe resaltar que existen límites de residualidad máxima de plaguicidas en matrices como agua, frutas, verduras y leche, pero no en sangre. Esto es muy llamativo debido a que las personas pueden incorporar estos compuestos desde diferentes fuentes como los alimentos, el ambiente (aire, agua), entre otros, y almacenarlos en el organismo en fuentes ricas en lípidos como la sangre o el tejido adiposo. Es por esto que resulta interesante evaluar la concentración de los residuos encontrados para realizar una comparación con los valores permitidos por ley para diferentes matrices, y con algunos reportados en trabajos a nivel nacional e internacional.

Como se puede observar en los datos encontrados en este estudio, seis de los plaguicidas OCs tienen concentraciones por encima de $0.01 \mathrm{ppm}$, y uno de los más tóxicos (Beta-BHC) más de $0.1 \mathrm{ppm}$. Del mismo modo, se muestran concentraciones preocupantes de plaguicidas OFs en las muestras evaluadas, llegando a más de 5 ppm en uno de los casos. Es la primera vez que encontramos tan altos niveles de plaguicidas en una matriz biológica. Existen algunos reportes de residuos de plaguicidas en sangre a nivel nacional e internacional, ${ }^{(23-26)}$ sin embargo se evalúan pocos compuestos y los resultados encontrados son reportados en partes por billón (ppb), es decir, hay una diferencia de un orden de magnitud en comparación con los resultados obtenidos en este estudio, siendo nuestras muestras en su mayoría entre cien y mil veces más concentradas que los reportes publicados. Esto nos permite sugerir que la problemática de los plaguicidas crece día a día en el mundo y que a nivel nacional existe un uso desmesurado de forma continua. Si bien son muchos los tipos de plaguicidas que se pueden evaluar, se encontraron algunas coincidencias con otros reportes nacionales (Tabla 2). Asimismo, se incluyen en la tabla algunos límites máximos de residuos (LMR) establecidos por entidades internacionales. Cabe resaltar que a la fecha no hay LMR de todos los alimentos $\mathrm{y}$ todos los plaguicidas consumidos y empleados en el mundo; en la base de datos solo se encuentran datos de los principales alimentos que influyen en la dieta diaria de la población mundial.

Como se puede observar en la Tabla 3, solo se enlistan cuatro de los 21 residuos encontrados en este estudio, los cuales coincidieron con un reporte nacional en condiciones similares sobre trabajadores agrícolas de un cultivo de flores. Asimismo, se comparan estas concentraciones con los LMR permitidos y establecidos por entidades internacionales que aplican a nivel global en algunos de los alimentos consumidos por la población, esto teniendo en cuenta que no existe una base de datos para suero humano.

Tabla 3. Residuos de plaguicidas encontrados en sangre. Comparación con otros autores y con los LMR establecidos por el Codex Alimentarius en algunos alimentos básicos ${ }^{27}$

\begin{tabular}{lccccc}
\hline Plaguicidas & $\begin{array}{c}\text { Máxima concentración } \\
\text { encontrada (ppm) }\end{array}$ & $\begin{array}{c}\text { Máxima concentración encontra- } \\
\mathbf{d a}(\mathrm{ppm}) \text { por otros autores }\end{array}$ & $\begin{array}{c}\text { LMR } \\
\text { frutas y } \\
\text { vegetales }\end{array}$ & $\begin{array}{c}\text { LMR } \\
\text { cereales }\end{array}$ & $\begin{array}{c}\text { LMR } \\
\text { leche }\end{array}$ \\
\hline $\begin{array}{l}\text { Heptacloro } \\
\text { epóxido }\end{array}$ & 0.009 & 0.02 & 0.01 & 0.02 & 0.006 \\
DDE & 0.003 & 0.02 & - & - & - \\
Aldrín & 0.031 & 0.019 & 0.05 & 0.02 & 0.006 \\
Dieldrín & 0.009 & 0.003 & 0.05 & 0.02 & 0.006 \\
\hline
\end{tabular}


Con respecto al heptacloro epóxido y DDE, se puede ver cómo nuestros resultados están por debajo de los reportados por otros autores; sin embargo esto se revierte en aldrín y dieldrín donde la concentración es casi el doble y el triple, respectivamente. Esto es muy relativo teniendo en cuenta que los cultivos fumigados son diferentes, lo que conlleva a diferentes tipos de plagas y consecuentemente diferentes plaguicidas. Lo único en lo que convergen los estudios es que evidentemente se genera residualidad en las personas que aplican estos compuestos. Al comparar los niveles encontrados en sangre con algunos alimentos básicos, se puede observar cómo en una matriz rica en grasa, como la leche, los LMR disminuyen considerablemente. En este sentido son preocupantes las concentraciones encontradas en sangre en un entorno lipídico donde los plaguicidas organoclorados como los mostrados en la Tabla 3, se estabilizan. Los plaguicidas OCs encontrados son derivados del lindano, causante de miles de enfermedades y muertes a nivel mundial, incluso se han detectado residuos de este fitosanitario y/o sus metabolitos en diversas matrices como leche y orina. ${ }^{28}$ De hecho, desde hace varias décadas se conoce su acción citotóxica, cancerígena y mutagénica. ${ }^{29,30,28} \mathrm{El}$ lindano y sus derivados alteran el funcionamiento de los canales de cloruro en las membranas nerviosas, interrumpiendo la transferencia de iones y la transmisión de impulsos entre células, estimulando al sistema nervioso central causando ataxia, convulsiones y muerte. ${ }^{31}$

Como se mencionó anteriormente, no existe una normativa que establezca los límites de residuos máximos de plaguicidas en sangre humana, lo cual surge como una necesidad debido a su gran repercusión sobre la salud pública. Estos resultados despiertan preocupación ya que son concentraciones altas de plaguicidas que se traducen en intoxicaciones crónicas o agudas en la población rural.

\section{DISCUSIÓN}

Desafortunadamente no existe en Colombia una legislación que establezca límites máxi- mos permitidos en el cuerpo humano; sin embargo, para tener una idea del nivel de contaminación que presentan las personas evaluadas es necesario comparar con los límites establecidos a nivel internacional para alimentos de origen vegetal o animal. Todos los alimentos destinados al consumo humano o animal en la Unión Europea (UE) están sujetos a un límite máximo de residuos de plaguicidas (LMR) en su composición, con el fin de proteger la salud humana. Dichos límites comprenden, por una parte, LMR específicos para ciertos alimentos destinados a las personas o los animales y, por otra, un límite general aplicable cuando no se haya fijado ningún LMR. ${ }^{32}$ El contenido máximo de residuos de plaguicidas en los alimentos es $0.01 \mathrm{ppm}$ o $\mathrm{mg} / \mathrm{kg}$. Este límite general es aplicable "por defecto", es decir, en todos los casos en que no se haya fijado un LMR de forma específica para un producto o un tipo de producto. Teniendo esto en cuenta y comparando los resultados de la Tabla 1, más del 50\% de los plaguicidas fueron encontrados por encima de los niveles máximos permitidos a nivel internacional en alimentos para consumo humano. Por lo tanto estas concentraciones encontradas son aún más alarmantes debido a que ya están presentes en el torrente sanguíneo. Cabe resaltar que el beta-BHD, heptacloro epóxido, endosulfan y Aldrinson son plaguicidas categorizados de alta toxicidad, que generan grandes efectos en la salud pública y en el medio ambiente. ${ }^{33}$ Asimismo, a excepción del 4,4-DDE, todos los plaguicidas de la Tabla 1 están prohibidos por la legislación nacional debido a su alta toxicidad y sin embargo son utilizados con frecuencia en las prácticas agrícolas. ${ }^{34,35}$ Lo mismo pasa con el metilparation, paration y thionazin, prohibidos por ley, que son altamente tóxicos y usados con frecuencia en cultivos regionales. ${ }^{34}$ La mayoría de los plaguicidas OFs, además de una intoxicación colinérgica, una toxicidad directa y un síndrome intermedio, son capaces de provocar una neuropatía de aparición retardada, la cual se presenta después de la fase aguda de las intoxicaciones, alrededor de la segunda y la cuarta semana, o tras un periodo indeterminado de exposición crónica. 
Esto se debe a la fosforilación de enzimas que afectan fundamentalmente los nervios periféricos, evolucionando hasta afectar el sistema nervioso central. ${ }^{36,37}$

Con respecto a las personas evaluadas, según las encuestas más del $75 \%$ comprenden edades entre 30 y 80 años, la mayoría realiza de manera inadecuada las prácticas agrícolas y según las observaciones realizadas, es clara la ausencia o el uso inadecuado de elementos de protección personal. Estos resultados son preocupantes y evidencian la necesidad de educar sobre el correcto uso de estos plaguicidas en los cultivos y de elementos de protección personal. Los trabajadores a los cuales se les realizó el estudio son personas con el mínimo de escolaridad, una edad media de $45 \pm 15.8$ años, y en su mayoría personas cabeza de familia.

Al evaluar el estudio a modo general, se comprueba la necesidad de implementar normas de calidad que impidan el uso de estos compuestos, o en su defecto reforzar los programas actuales de control, vigilancia y monitoreo de plaguicidas en alimentos y aplicadores. Asimismo, generar nuevos métodos de control de plagas que no representen peligro para la salud humana, ya que no solo afecta a los aplicadores, sino también a sus familias, al ecosistema que los rodea y a los consumidores que ingieren las frutas y vegetales fumigadas. Los resultados del estudio demuestran que existen aspersiones excesivas y uso de productos no permitidos en los principales alimentos consumidos por la población regional; del mismo modo se evidencia una fuerte tendencia a utilizar un amplio abanico de diferentes productos en un solo cultivo, sin tener en cuenta las buenas prácticas agrícolas. Estos resultados comprueban el alto riesgo al que se exponen constantemente las personas que aplican plaguicidas y señalan la importancia de continuar con los estudios de monitoreo y control de calidad de los principales alimentos, fuentes hídricas y trabajadores agrícolas, para informar a la población de los riesgos a los que está expuesta y de este modo impulsar un mejoramiento de las normas actualmente establecidas por los sectores de vigilancia.

\section{AGRADECIMIENTOS}

Los autores agradecen a la Vicerrectoría de Investigaciones de la Universidad del Quindío por el apoyo financiero para la realización de este trabajo. Asimismo a los trabajadores agrícolas por su buena voluntad para la toma de muestras.

\section{REFERENCIAS}

1. Issa Y, Sham F, Nijem K, Bjertness E, Kristensen P. Pesticide use and opportunities of exposure among farmers and their families: cross-sectional studies 1998-2006 from Hebron governorate, occupied Palestinian territory. Environ Health. 2010; 9: 63-72.

2. Barr D, Ananth C, Yan X, Lashley S, Smulian JC, Ledoux TA et al. Pesticide concentrations in maternal and umbilical cords era and their relation to birth out comes in a population of pregnant women and newborns in New Jersey. Sci Total Environ. 2010; 408(4): 790-795.

3. Hodgson E, Wallace AD. Human Metabolic Interactions of Pesticides: Inhibition, Induction, and Activation, Parameters for Pesticide QSAR and PBPK/PD Models for Human Risk Assessment. ACS Symposium Series. Carpet 8. Washington DC: American Chemical Society. 2012, pp. 115-132.

4. Richard S, Moslemi S, Sipahutar H, Benachour N, Seralini GE. Differential effects of glyphosate and round up on human placental cells and aromatase. Environ Health Perspect. 2005; 113: 716-720.

5. Charlier C, Plomteux G. Environmental chemicals pollution and risk of human exposure: the role of organochlorine pesticides. Ann Biol Clin. 2002; 60:37-46.

6. Waliszewski SM, Infanzon RM, Hart MM. Differences in persistent organochlorinepesticides concentrations between breast adipose tissue and blood serum. Bull Environ Contam Toxicol. 2003; 70: 920-926.

7. Colombia. Instituto Nacional de Salud. Subdirección de Vigilancia y Control en Salud Pública. Grupo Factores de Riesgo Ambiental. Informe de intoxicaciones por 
plaguicidas [en línea]. Colombia: SIVIGILA; 2010 [consultado mayo de 2011]. Disponible en: http://www.minsalud.gov.co/ comunicadosPrensa/Documents/INTOXICACION_POR_PLAGUICIDAS.pdf

8. SENA - ANDI. Uso adecuado y eficaz de productos para la protección de cultivos. En: Memorias por: Alfredo Ramos Ángel, Campaña de prevención, $4^{\text {a }}$ ed. Colombia: Ed. Produmedios; 2005. pp. 13-233.

9. Olsson AO, Baker SE, Nguyen JV et al. A liquid chromatography-Tandem Mass Spectrometry Multiresidue Method for Quantification of Specific Metabolites of Organophosphorus Pesticides, Synthetic Pyrethroids, Selected Herbicides, and DEET in Human Urine. Anal Chem. 2004; 76: 2453-2461.

10. Plenge F, Sierra JA, Castillo YA. Riesgos a la salud humana causados por plaguicidas. Tecnociencia Chihuahua. 2007; 1(3): 4-6.

11. Monroy CM, Cortés AC, Sicard DM, De Restrepo HG. Cytotoxicity and genotoxicity of human cells. Exposed in vitro to glyphosate. Biomédica. 2005; 25: 335-345.

12. Dalvie M, Cairncross E, Solomon A, London L. Contamination of rural surface and ground water by endosulfan in farming areas of the Western Cape, South Africa. Environ. Health. 2003; 2: 1-15.

13. Martínez A, Reyes I, Reyes N. Cytotoxicity of the herbicide glyphosate in human peripheral blood mononuclear cells. Biomédica. 2007; 27(4): 594-604.

14. Gutiérrez JA, Pinzón MI, Londoño A, Blach D, Rojas AM. Residuos de plaguicidas organoclorados, organofosforados y análisis fisicoquímico en piña (Ananascomosus L.). Agro Sur. 2010; 38(3): 199-211.

15. Focant JF, Sjodin A, Turner WE, Patterson JRDG. Measurement of Selected Polybrominated Diphenyl Ethers, Polybrominated and Polychlorinated Biphenyls, and Organochlorine Pesticides in Human Serum and Milk Using Comprehensive Two-Dimensional Gas ChromatographyIsotope Dilution Time-of-Flight Mass Spectrometry. Anal Chem. 2004; 76: 6313-6320.

16. Gutiérrez JA, Londoño A. Determinación de plaguicidas organoclorados y organofos- forados en tomates de mercados de cadena en las ciudades de Pereira y Armenia, Colombia. BLACPMA. 2009; 8(3): 165-171.

17. Guan H, Brewer W, Garris ST, Craft SC, Morgan LS. Multiresidue Analysis of Pesticides in Fruits and Vegetables Using Disposable Pipette Extraction (DPX) and Micro-Luke Method. J Agric Food Chem. 2010; 58: 5973-5981.

18. Londoño A, Arrubla JP, Zárate M, Torres D, Beltrán M, Toro JL, Morales IT. Determinación de la calidad ambiental de la quebrada Agua Bonita, corregimiento de Barcelona, departamento del Quindío. Revista de investigaciones Universidad del Quindio. 2005; 15: 55-64.

19. Londoño A, Zárate M, Torres D et al. Determinación de la calidad ambiental del río El Roble cuenca media y baja en el departamento del Quindío. Revista de la Asociación Nacional de Ciencias Biológicas. 2005; 229-229.

20. Norris EH, Uryu K, Leight S, Giasson BI, Trojanowski JQ, Lee VMY. Pesticide Exposure Exacerbates $\alpha$-Synucleinopathy in an A53T Transgenic Mouse Model. The Am J Pathol. 2007; 70(2): 658-666.

21. McKinlay R, Plant JA, Bell JNB, Voulvoulis N. Endocrine disrupting pesticides: Implications for risk assessment. Environ Int. 2008; 34(2): 168-183.

22. Ueno E, Oshima H, Saito I, Matsumoto H. Determination of nitrogen- and phosphoruscontaining pesticide residues in vegetables by gas chromatography with nitrogen-phosphorus and flame photometric detection after gel permeation chromatography and a two-step mini column clean up. J AOAC Int. 2003; 86: 1241-1251.

23. Hunter RE Jr, Riederer AM, Ryan PR. Method for the determination of organophosphorus and pyrethroid pesticides in food via gas chromatography with electroncapture detection. J Agric Food Chem. 2010; 58:1396-1402.

24. López R, Goñi F, Etxandia A, Millán E. Determination of organochlorine pesticides and polychlorinated biphenyls in human serum using head space solid-phase micro extraction and gas chromatographyelectron capture detection. J Chromatogr B 
Analyt Technol Biomed Life Sci. 2007 Feb 1; 846(1-2): 298-305.

25. Volz SA, Johnston JJ, Griffin DL. Solid phase extraction gas chromatography/electron capture detector method for the determination of organochlorine pesticides in wild life whole blood. J Agric Food Chem. 2001; 49: 2741-2745.

26. López FJ, Pitarch E, Egea S, Beltran J, Hernández F. Gas chromatographic determination of organochlorine and organophosphorus pesticides in human fluids using solid phase microextraction. Anal Chim Acta. 2001; 433: 217-226.

27. U.S. Department of Health and Human Services. Agency for Toxic Substances and Disease Registry. Toxicological profile for alpha, beta, gamma, and delta-hexachlorocyclohexane [en línea]. Atlanta: Agency for Toxic Substances and Disease Registry; 2005 [consultado febrero de 2013]. Disponible en: http://www.atsdr. cdc.gov/toxprofiles/tp43.pdf

28. Richardson JR, Shalat SL, Buckley B et al. Elevated serum pesticide levels and risk of Parkinson disease. Arch Neurol. 2009; 66(7): 870-875.

29. US Environmental Protection Agency. Reregistration Eligibility Decision for Endosulfan [en línea]; US: EPA; 2002. [consultado octubre de 2013]. Disponible en: http://www.epa.gov/oppsrrd1/REDs/endosulfan_red.pdf

30. U.S. Department of Health and Human Services, Agency for Toxic Substances and Disease Registry. Draft Toxicological Profile for Endosulfan [Consultado diciembre de 2013]; Atlanta: U.S. Department of Health and Human Services; 2003. Disponible en: http://www.atsdr.cdc.gov/toxprofiles/tp41.pdf

31. Morowati M. Inhalation toxicity studies of thimet (phorate) in male Swiss albino mouse, Mus musculus: I. Hepatotoxicity. Environ Pollut. 1997; 96(3): 283-288.

32. Soreq H, Seidman S. Acetylcholinesterase, new roles for an old actor. Na. Rev Neurosci. 2001; 2(4): 294-302.

33. Vargas Melo A, Vallejor MC. Cuantificación de insecticidas organoclorados en sangre. Rev Fac Med [en línea]: 71-85 [Consultado mayo de 2014]. Disponible en: http://www. bdigital.unal.edu.co/24727/1/21934-752181-PB.PDF

34. Pitarch Arquimbau E. Desarrollo de metodología analítica para la determinación de plaguicidas organofosforados y organoclorados en muestras biológicas humanas [en línea]. Tesis doctoral. España: Universitat Jaume; 2001 [consultado en mayo 2014]. Disponible en: http://repositori.uji.es/ xmlui/handle/10234/29541

35. Volz S A, Johnston JJ, Griffin DL. Solid phase extraction gas chromatography/electron capture detector method for the determination of organochlorine pesticides in wild life whole blood. J Agric Food Chem. 2001; 49: 2741-2745.

36. 36. Focant JF, Sjodin A, Turner WE, Patterson DG. Measurement of Selected Polybrominated Diphenyl Ethers, Polybrominated and Polychlorinated Biphenyls, and Organochlorine Pesticides in Human Serum and Milk Using Comprehensive Two-Dimensional Gas ChromatographyIsotope Dilution Time-of-Flight Mass Spectrometry. Anal Chem. 2004; 76: 6313-6320.

37. Pesticide Maximum Residue Limits. Codex Alimentarius [Consultado mayo de 2014]. Disponible en: http://www.codexalimentarius.net/pestres/data/index.html

38. Schafer K. Going, Going, Gone: Lindane moves closer to elimination. Global Pesticide Campaigner. San Francisco, CA. 2000.

39. Tsushimoto G, Chang CC, Trosko JE, Matsumura F. Cytotoxic, mutagenic, and cellcellcommunication inhibitory properties of DDT, lindane, and chlordane on Chinesehamster cells in vitro. Arch Environ Contam Toxicol. 1983; 12(6): 721-730.

40. Wolffe M, Toniolo PG, Lee EW, Rivera M, Dubin N. Blood levels of organochlorine residues and risk of breast cancer. Je Nat Cancer Ins. 1993; 855(8): 648-652.

41. U.S. Department Of Health And Human Services. Public Health Service. Agency for Toxic Substances and Disease Registry. Toxicological Profile For Alpha-, Beta-, Gamma-, and Delta-Hexachlorocyclohexane [en línea]. Atlanta: US Department Of Health And Human Services; 
2005 [consultado 8.05.2014]. Disponible en: http://www.atsdr.cdc.gov/toxprofiles/ tp43.pdf

42. Union Europea. Regulation (EC) No 396/2005 of the European Parliament. Europeanmaximumpesticideresidues [en línea]. [actualizado 4.04.2011; consultado diciembre de 2013]. Disponible en: http://europa.eu/legislation_summaries/food_safety/ plant_health_checks/121289_es.htm

43. Organización Mundial de la Salud; Organización Panamericana de la Salud. División Salud y Ambiente. Plaguicidas y Salud en las Américas [en línea] Washington; 1993. [consultado octubre de 2013]. Disponible en: http://www. minsalud.gov.co/comunicadosprensa/documents/intoxicacion_por_plaguicidas.pdf

44. Instituto Colombiano Agropecuario ICA. Resoluciones 447/74, 209/78, 2471/91 y 1311/01. Restricciones, prohibiciones y suspensión de registros de plaguicidas de uso agrícola en Colombia [en línea]. [consultado febrero de 2013]. Disponible en: http://www. ica.gov.co/getdoc/b2e5ff99-bd80-45e8-aa7a$55 \mathrm{fob} 5 \mathrm{~b} 42 \mathrm{dc} /$ plaguicidas-rohibidos.aspx

45. Colombia. Ministerio de Salud. Resolución 10255 Restricciones, prohibiciones y suspensión de registros de plaguicidas de uso agrícola en Colombia [en línea] 1993. [consultado febrero de 2013]. Disponible en: http://www.ica.gov.co/getdoc/b2e5ff99bd80-45e8-aa7a-e55f0b5b42dc/PLAGUICIDAS-ROHIBIDOS.aspx

46. Johnson MK. Delayed neurotoxicity induced by organophosphorus compounds. Areas of understanding and ignorance. Dev Toxicol Environ Sci. 1980; 8: 27-38.

47. Yelamos F, Diez F, Martin JC et al. Intoxicaciones agudas por insecticidas organofosforados en la provincia de Almería. Estudio de 187 casos. Med. Clin. (Barc.) 1992; 98: 681-684. 